\title{
Predictors of Functional Outcome after Emergency Carotid Artery Stenting and Intra-Arterial Thrombolysis for Treatment of Acute Stroke Associated with Obstruction of the Proximal Internal Carotid Artery and Tandem Downstream Occlusion
}

H.S. Kwak, S.B. Hwang, G.Y. Jin, D.S. Hippe, and G.H. Chung

\begin{abstract}
BACKGROUND AND PURPOSE: Patients who develop severe stroke symptoms due to acute internal carotid artery occlusion eventually in combination with a thromboembolic obstruction of the middle cerebral artery incur a major risk of developing extensive MCA infarction with a poor outcome. The purpose of this study was to evaluate the outcome for patients with tandem occlusions in the MCA and/or distal ICA, retrospectively, who had undergone stent implantation in the proximal segment of the ICA in addition to intra-arterial thrombolysis.
\end{abstract}

MATERIALS AND METHODS: Thirty-five patients with tandem occlusions of the MCA and/or distal ICA and acute occlusion of the proximal ICA underwent stent implantation for the proximal ICA occlusion and IAT for the tandem occlusion. Clinical outcome measures were assessed on admission and at discharge by using the National Institutes of Health Stroke Scale as well as 3 months after treatment by using the modified Rankin Scale.

RESULTS: The median NIHSS score on admission was 12 (range, 6-22). All patients had patent flow into the M1 and ICA after carotid artery stent placement and IAT. After the procedure, 19 patients (54.3\%) were $\mathrm{TICl}$ grade III; 7 (20.0\%), TICl grade IIb; and 9 (25.7\%), TICI grade Ila. Symptomatic intracerebral hemorrhage occurred in 1 patient (2.9\%). The overall mortality rate was 11.4\% (4/35). At 3-month follow-up, the median NIHSS score was 4 (range, 1-17). NIHSS score at admission and $\mathrm{TICl}$ grade were all found to be independently associated with an unfavorable outcome at 3 months.

CONCLUSIONS: Initial stroke severity, degree of successful revascularization, and the side of ischemia were found to independently predict the functional outcome at 3 months after treatment.

ABBREVIATIONS: CAS = carotid artery stenting; IAT = intra-arterial thrombolysis; $\mathrm{mRS}=$ modified Rankin Scale; $\mathrm{TICI}=$ Thrombolysis in Cerebral Ischemia; $\mathrm{UK}=$ urokinase

O

cclusion of the carotid artery is clinically silent or accompanied by only mild symptoms if there is adequate collateral flow. ${ }^{1}$ However, there is a subgroup of patients who develop severe stroke symptoms due to acute internal carotid artery occlusion eventually in combination with a thromboembolic obstruction of the central arteries, such as the terminal carotid segment or the middle cerebral artery, and subsequently incur a major risk of developing extensive MCA infarction with a poor outcome. ${ }^{2,3}$ In this group of patients, early restoration of flow in the occluded ICA may

Received March 21, 2012; accepted after revision June 27.

From the Department of Radiology and Research Institute of Medical Science (H.S.K., S.B.H., G.Y.J., G.H.C.), Chonbuk National University Medical School and Hospital, Chonbuk, South Korea; and Department of Radiology (D.S.H.), University of Washington, Seattle, Washington.

Please address correspondence to Hyo-Sung Kwak, MD, Department of Radiology and Research Institute of Medical Science, Chonbuk National University Medical School and Hospital, 634-18, Keumam-Dong, Jeonju-shi, Chonbuk, 561-712, South Korea; e-mail: kwak8140@jbnu.ac.kr

--m Indicates open access to non-subscribers at www.ajnr.org

http://dx.doi.org/10.3174/ajnr.A3304 improve the symptoms of acute stroke, prevent stroke deterioration, and reduce the long-term stroke recurrence risk. Some authors have reported successful revascularization of patients with acute stroke symptoms secondary to ICA occlusions. ${ }^{4-8}$ However, previous studies have some potential drawbacks: 1) a broad range of time windows between symptom onset and treatment, 2) a small number of cases, 3) a small number of tandem lesions into the MCA and distal ICA, and 4) minor or mild neurologic deficits. Therefore, the purpose of our study was to evaluate retrospectively the outcome for patients with tandem lesions in the MCA and/or distal ICA who had undergone stent implantation in the proximal segment of the ICA in addition to intra-arterial thrombolysis.

\section{MATERIALS AND METHODS \\ Patient Selection}

This retrospective analysis was conducted with institutional review board approval. Between 2005 and 2010, patients with acute stroke symptoms who were admitted to our university-based 
stroke center within 6 hours of symptom onset were considered for inclusion in this study. The diagnosis of ischemic stroke was based on the presence of a focal neurologic deficit with or without demonstration of corresponding ischemic lesions. Permission for pharmacologic or mechanical thrombolytic treatment, including carotid artery stent placement and IAT, was obtained from the patient's legal representatives. The inclusion criteria for this study were the following: 1) occlusion of the ipsilateral proximal ICA, confirmed by angiography; 2) the presence of a tandem thrombotic lesion with acute complete obstruction of the distal ICA or MCA (M1 and/or M2), confirmed by intra-arterial digital subtraction angiography; 3) anterior circulation stroke symptoms within 6 hours before treatment; 4) CT or MR imaging exclusion of hemorrhage; and 5) CT signs of ischemia that affected less than one-third of the MCA territory. Angiography was performed by 1 interventional neuroradiologist with 5 years of experience in endovascular treatment.

\section{Endovascular Treatment}

On admission, the National Institutes of Health Stroke Scale score was assessed by a stroke neurologist. Eligible patients who met standard National Institute of Neurologic Disorders and Stroke criteria were treated with $0.9 \mathrm{mg} / \mathrm{kg}$ of IV rtPA.

Endovascular treatment was performed by the same interventional neuroradiologist. Routine 3- or 4-vessel cerebral angiography was performed before treatment for evaluation of collateral flow. Then the double coaxial system, which was assembled by combining an outermost 80 -cm-long $8 \mathrm{~F}$ introducer sheath (Super Arrow Flex; Arrow International, Reading, Pennsylvania) and an inner 100-cm-long 8F guiding catheter (Guider Softip; Boston Scientific, Natick, Massachusetts), was placed in the common carotid artery to enable placement of the carotid artery stent and the PS reperfusion catheter. Systemic anticoagulation was initiated with a 3000-U bolus of intravenous heparin followed by 1000$\mathrm{U} / \mathrm{hr}$ infusion. An end-hole microcatheter (Microferret; Cook, Bjaeverskov, Denmark) over a straight microguidewire (Synchro14 ; Boston Scientific) was advanced through an $8 \mathrm{~F}$ guiding catheter. After complete occlusion was demonstrated, a microguidewire was gently passed into the ipsilateral common carotid artery, through the occlusion, and then into the distal cervical ICA. Repeat angiography of the ICA distal to the occlusion through microcatheter was performed to assess thrombus/occlusion in the intracranial circulation (tandem occlusion).

All patients included in this study had thrombus in the MCA and/or distal ICA segment. An embolic protection device was not used for any of these patients. The microcatheter was exchanged for a small-diameter $(3.0-\mathrm{mm})$ angioplasty balloon, which was inflated to 6 atm to predilate the occlusion. Deployment of a selfexpandable stent (Precise; Cordis, Miami Lakes, Florida), the size of which was chosen according to the presumed parent size, was performed over the wire. Poststenting angioplasty by using a 5- to 6-mm diameter balloon was performed to achieve a residual diameter stenosis of $<20 \%$.

After CAS, cerebral angiography was performed for evaluation of occlusion of the MCA and/or the distal ICA. An end-hole microcatheter was advanced over a microguidewire through an $8 \mathrm{~F}$ guide catheter into the occlusion site in patients with M1, M2, or distal ICA thrombus. The microcatheter tip was placed into the thrombus, and then a 100,000-U bolus of urokinase diluted to 10 $\mathrm{mL}$ in a mixture of saline and contrast medium (7:3) was manually infused for 3-5 minutes. We mixed the contrast medium for early detection of recanalization during UK infusion. We performed the repeat UK injection and mechanical clot disruption with a microcatheter and a microguidewire after cerebral angiography for evaluation of recanalization of the occluded vessel. Mechanical clot disruption was undertaken after 100,000 U of UK was administered. Mechanical clot maceration consisted of multiple passes of the microguidewire through the clot. The microguidewire was gently rotated clockwise, while being advanced. During this process, the microcatheter was often advanced multiple times over the microguidewire as well. After withdrawal of the microguidewire from the microcatheter, an additional 100,000 $\mathrm{U}$ of UK was manually infused for 3-5 minutes through the microcatheter at the site of the remaining thrombus. The UK infusions were stopped immediately if the control angiograms showed complete recanalization.

Mechanical clot disruption by using a microcatheter and a microguidewire was reattempted if recanalization was not achieved after UK infusion. One patient with distal migration of thrombus into the posterior cerebral artery was additionally infused with 100,000 U of UK through a microcatheter at the site of the occluded artery. The patients who did not achieve complete recanalization after mechanical clot disruption by using a microcatheter and a UK infusion underwent percutaneous angioplasty. Patients with distal ICA thrombus underwent the combined procedure, such as aspiration thrombectomy with a $5 \mathrm{~F}$ catheter and/or reperfusion catheter, UK injection, and mechanical clot disruption, with a microcatheter and microguidewire.

Each patient was given an oral load of clopidogrel (300 mg) immediately after the procedure, and each received maintenance doses of both aspirin ( $100 \mathrm{mg}$ daily) and clopidogrel ( $75 \mathrm{mg}$ daily) starting the day after the procedure. In addition, strict blood pressure guidelines were adhered to by maintaining a mean blood pressure of $<100 \mathrm{mg}$ for at least 48 hours after revascularization in the Neuro Intensive Care Unit because of concerns of hyperperfusion to the ipsilateral hemisphere.

\section{Data Collection}

Pretreatment and posttreatment angiograms were evaluated by the same interventional neuroradiologist. The recanalization status was classified according to the Thrombolysis in Cerebral Ischemia perfusion scale. TICI perfusion categories include grade 0 (no perfusion), grade I (penetration with minimal perfusion), grade II (partial perfusion), grade IIa (partial perfusion with incomplete distal filling of $<50 \%$ of the expected territory), grade IIb (partial perfusion with incomplete distal filling of 50\%-99\% of the expected territory), and grade III (full perfusion with normal filling of the distal branches in a normal hemodynamic fashion). Recanalization was defined as TICI grade II or III.

For each patient, age, sex, NIHSS score on admission, time from symptoms to IA therapy, duration of the procedure, UK dose, recanalization status, occurrence of symptomatic intracranial hemorrhage, and modified Rankin Scale score at discharge were recorded. After 3 months, the patients were brought back for 
another clinical evaluation and updated NIHSS and mRS scores were recorded. The clinical evaluation was performed by stroke neurologists who were not blinded to the treatment. The primary clinical outcome was the mRS at 3 months, which was further dichotomized as favorable $(\mathrm{mRS} \leq 2)$ or unfavorable $(\mathrm{mRS}>2)$.

\section{Statistical Analysis}

Continuous values are expressed as the interquartile range and/or the range. Categoric variables are summarized by using counts and percentages. Patients were grouped by favorable ( $\mathrm{mRS} \leq 2)$ and unfavorable (mRS $>2$ ) outcomes at 3 months. Continuous and categoric variables were compared between these groups by using the Mann-Whitney $U$ test and Fisher exact test, respectively. Linear trends between favorable/unfavorable outcome and TICI grade were tested by using the $\chi^{2}$ test for trend. Multivariate analysis for favorable/unfavorable outcome was conducted by using logistic regression. Variables with $P<.20$ from univariate analysis were taken as candidate predictors and were entered into a backward-selection algorithm to identify a set of independent predictors. Statistical significance was defined as $P<.05$. All statistical analysis was performed by using R 2.14.1 (R Foundation for Statistical Computing, Vienna, Austria).

\section{RESULTS}

Between 2005 and 2010, two hundred fifty-four consecutive patients with acute ischemic stroke were admitted to our institution within 6 hours of onset of symptoms. Of these patients, 42 were confirmed to have proximal ICA occlusion, tandem thrombotic distal ICA/MCA occlusion, and ischemia affecting less than onethird of the MCA territory by CT. Five patients did not undergo recanalization of the ICA obstruction either because there was good or moderate leptomeningeal collateral flow and/or contralateral collateral flow to the territory of the occluded MCA $(n=$ 3 ) or because CT showed that the ischemia affected more than one-third of the MCA territory $(n=2)$. Two patients were lost to follow-up and did not receive a clinical evaluation at 3 months, leaving 35 patients available for analysis.

Baseline clinical characteristics and preprocedural findings are summarized in Table 1. Most patients were men (88.6\%) with ages between 51 and 84 years (median, 65 years). Six (17.1\%) had a medical history of stroke. The time from symptom onset to admission ranged from 60 to 340 minutes (median, 180 minutes), and the time from onset to IAT ranged from 140 to 435 minutes (median, 270). The median NIHSS score at admission was 12 (range, 6-22). Eight patients (22.9\%) received IV rtPA before angiography. All patients had a tandem thrombotic occlusion distal to the primary ICA occlusion. Twenty-five (71.4\%) patients had a tandem intracranial occlusion in M1, and 5 (14.3\%) had an occlusion in M2. The remaining 5 had an occlusion of distal ICA.

Table 1 also summarizes the clinical findings during and after the interventional procedure. The technical success rate of the carotid artery stent placement after passage of the microguidewire was $100 \%$. The median duration of IA therapy was 90 minutes (range, 40-180 minutes). The total UK dose used most was frequently $100,000 \mathrm{U}$, but the dose ranged up to 500,000 $\mathrm{U}$ in some cases. All patients had patent flow into the M1 and ICA after carotid artery stent placement and IAT (TICI grade II or III). After
Table 1: Summary of patient demographics, preprocedural findings, periprocedural findings, and outcomes after 3 months

\begin{tabular}{lcc}
\hline \multicolumn{1}{c}{ Patient Demographics } & $\begin{array}{c}\text { No. (\%) or } \\
\text { Median (IQR) }\end{array}$ & Range \\
\hline Age (yr) & $65(63-76)$ & $51-84$ \\
Male & $31(88.6)$ & - \\
Previous medical history & - & - \\
$\quad$ Diabetes mellitus & $7(20.0)$ & - \\
Hypertension & $19(54.3)$ & - \\
Hypercholesterolemia & $10(28.6)$ & - \\
Smoking & $17(48.6)$ & - \\
Cardiac disease & $5(14.3)$ & - \\
Prior stroke & $6(17.1)$ & -
\end{tabular}

\section{Preprocedural findings}

Time to hospital (min)

Time to IA therapy (min)

NIHSS at admission

Modified Rankin scale

3

4

5

Right-sided occlusion

Tandem occlusion site

M1

M2

Distal ICA thrombus

Received IV rtPA

Periprocedural findings

Procedure duration ( $\mathrm{min})$

Urokinase dose $\left(\times 10^{4} \mathrm{U}\right)$

$\mathrm{TICl}$ grade

Ila

Ilb

III

Complications

Symptomatic hemorrhage

Brain swelling

Follow-up evaluation at 3 months NIHSS

Modified Rankin Scale

0

1

2

3

4

5

6

Favorable outcome

Mortality

\begin{tabular}{|c|c|}
\hline 180 (125-210) & $60-340$ \\
\hline 270 (190-310) & $140-435$ \\
\hline 12 (8-17) & $6-22$ \\
\hline $4(3-4)$ & $3-5$ \\
\hline $11(31.4)$ & - \\
\hline $21(60.0)$ & - \\
\hline $3(8.6)$ & - \\
\hline 15 (42.9) & - \\
\hline - & - \\
\hline 25 (71.4) & - \\
\hline $5(14.3)$ & - \\
\hline $5(14.3)$ & - \\
\hline $8(22.9)$ & - \\
\hline $90(68-120)$ & 40-180 \\
\hline $10(10-30)$ & $10-50$ \\
\hline - & - \\
\hline $9(25.7)$ & - \\
\hline $7(20.0)$ & - \\
\hline $19(54.3)$ & - \\
\hline $2(5.7)$ & - \\
\hline $1(2.9)$ & - \\
\hline $1(2.9)$ & - \\
\hline $4(2-7)$ & $1-17$ \\
\hline $2(1-3)$ & $0-6$ \\
\hline $3(8.6)$ & - \\
\hline $11(31.4)$ & - \\
\hline $8(22.9)$ & - \\
\hline $5(14.3)$ & - \\
\hline $4(11.4)$ & - \\
\hline $0(0.0)$ & - \\
\hline $4(11.4)$ & - \\
\hline $22(62.9)$ & - \\
\hline $4(11.4)^{\prime}$ & - \\
\hline
\end{tabular}

Note:- IQR indicates interquartile range; IA indicates intra-arterial.

${ }^{\text {a }}$ From onset of symptoms.

${ }^{b}$ Excluding the 4 patients who died within 3 months. Favorable outcome was defined as a modified Rankin Scale score of $\leq 2$ at 3 months.

the procedure, 19 patients $(54.3 \%)$ were classified as TICI grade III, $7(20.0 \%)$ as TICI grade IIb, and $9(25.7 \%)$ as TICI grade IIa.

No procedure-related complications such as vessel rupture or dissection were observed. However, symptomatic intracerebral hemorrhage occurred in 1 patient $(2.9 \%)$ after the procedure. Another patient underwent decompressive surgery due to brain swelling and edema. Even though they received intravenous rtPA injections because of early admission after symptom onset, 2 patients had high initial NIHSS scores (20 and 22) and showed severe deterioration immediately after the procedure and later died. 

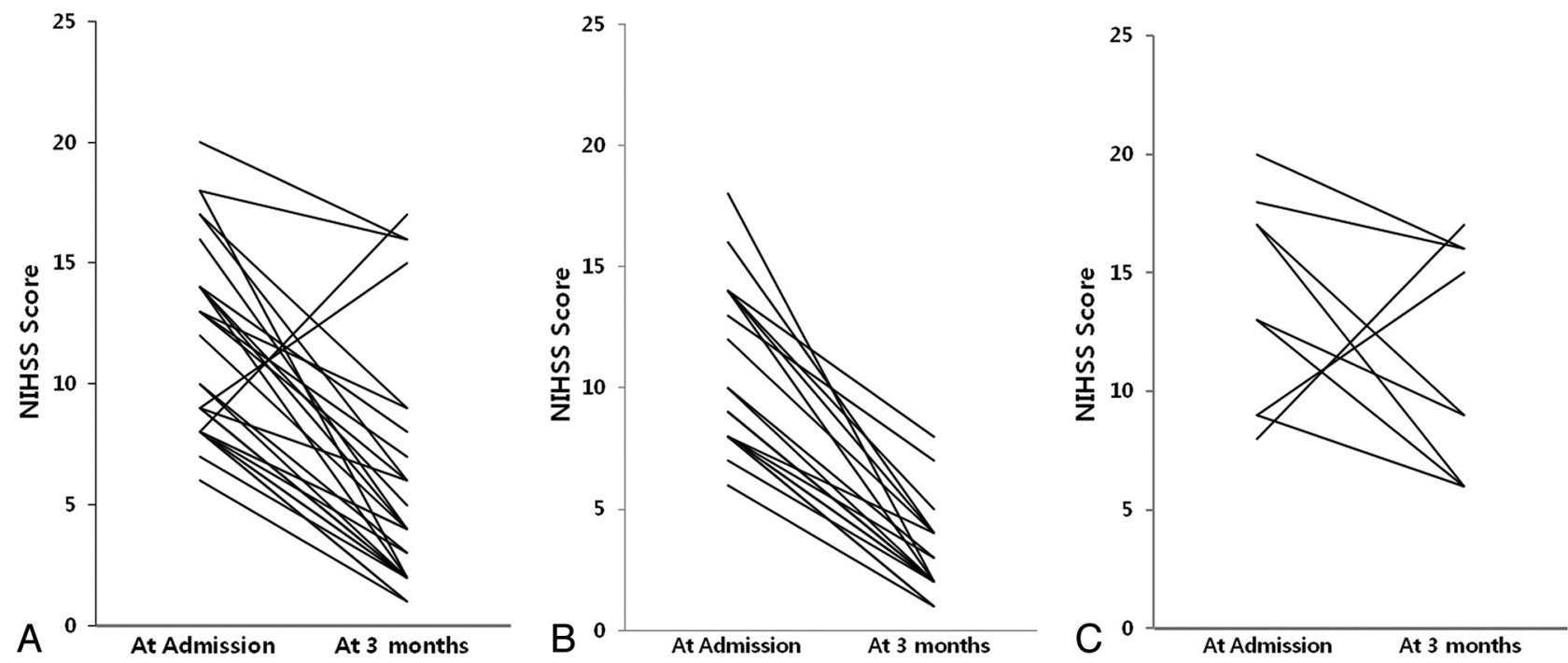

FIG 1. The NIHSS score on admission and at 3 months. A, NIHSS score of all patients $(n=31)$ excluding 4 patients who died. Median NIHSS scores were 12 on admission and 4 at 3 months ( 3 patients went from 9 to 2 , three patients went from 8 to 1 , and 2 patients went from 9 to 1 ). $B$, NIHSS scores of patients $(n=22)$ with favorable outcomes (mRS, $0-2)$ ( 3 patients went from 9 to 2 , three patients went from 8 to 1 , and 2 patients went from 9 to 1). Median NIHSS scores were 9 and 2. C, NIHSS scores of patients $(n=9)$ with unfavorable outcomes (mRS, $3-6)$. Median NIHSS scores were 17 and 9, respectively.

Another 2 patients who were neurologically stable after the procedure died 30 days later secondary to pneumonia and sepsis. Therefore, the overall mortality rate was $11.4 \%$.

At 3 months, the median NIHSS score of the patients who did not die was 4 (range, 1-17) (Fig 1). All except 2 demonstrated improved NIHSS scores at 3 months. However, 13 patients $(37.1 \%)$ had unfavorable functional outcomes (mRS $>2$, including the 4 who died). Table 2 compares the patient demographics, procedural findings, and follow-up evaluations between those with favorable and unfavorable outcomes. On average, those with unfavorable outcomes tended to be older $(P=.040)$, have higher initial NIHSS $(P=.003)$ and $\mathrm{mRS}(P=.005)$ scores, and have a right-sided occlusion $(P=.032)$ and a lower TICI grade $(P=$ .006). They also had higher NIHSS scores at 3 months.

Multivariate logistic regression was used to identify independent predictors of unfavorable functional outcome. A backwardselection algorithm was applied to the variables with $P<.20$ during univariate analysis. NIHSS scores at admission, TICI grades, and the presence of a right-sided occlusion were all found to be independently associated with unfavorable outcome at 3 months (Table 3). While very large odds ratios were seen for the latter 2, the confidence intervals were also quite wide, indicating substantial uncertainty as to the true magnitudes of the associations.

\section{DISCUSSION}

Many authors have reported the clinical usefulness of stent-assisted thrombolysis in patients with acute occlusion of the extracranial ICA. ${ }^{4-9}$ Emergency carotid artery stent placement is expected to reopen an extracranial ICA occlusion with reduction in cerebral blood flow in the affected hemisphere. Also, early flow restoration across the tandem lesion in the MCA and/or distal ICA after extracranial ICA stent placement would aim to reverse the ischemic process by stopping the expansion of the ischemic core into the penumbra. Because the size of the ischemic core is known to correlate with clinical outcome, this approach is expected to maximize the chances of a favorable outcome. ${ }^{10}$

Nedeltchev et $\mathrm{al}^{5}$ reported on 25 patients with acute stroke and acute occlusion of the proximal carotid artery who had been treated by pharmacomechanic lysis and stent implantation into the ICA. They were compared with a group of patients $(n=31)$ who had received heparinization and antiplatelet medication but in whom no recanalization of the carotid artery had been attempted. In the stented group, 5 of $25(20 \%)$ patients died, as did 5 of 31 in the medication group (16\%). The authors reported a favorable outcome in $56 \%$ of those in the recanalization group and in only $26 \%$ of those in the medication group. Although there was a relatively short follow-up duration in our study, 22 of 35 patients $(62.9 \%)$ patients showed favorable clinical outcomes $(\mathrm{mRS} \leqq 2)$.

Tandem obstruction of both the ICA and the MCA is frequently highly symptomatic, and only $20 \%$ of cases are associated with a good outcome ( $\mathrm{mRS} \leqq 2$ ) despite intravenous thrombolysis. ${ }^{10}$ Patients with tandem occlusion had worse short- and longterm clinical outcomes. Linfante et $\mathrm{al}^{11}$ found a recanalization rate of $31 \%$ with intravenous thrombolysis in patients with ICA occlusion compared with $88 \%$ in patients with MCA occlusion. Jovin et $\mathrm{al}^{4}$ reported that patients without a tandem occlusion were more likely to show rapid neurologic improvement within the first 24 hours after carotid artery stent placement and an $\mathrm{mRS} \leqq 2$ at follow-up. In our study, all patients had a tandem lesion in the MCA and/or distal ICA. Good outcomes ( $\mathrm{mRS} \leqq 2$ at 3 months) in our study were found in $62.9 \%$ of the patients compared with $56 \%$ of patients who reached an mRS score of $\leqq 2$ at 3 months in a previous study. ${ }^{5}$

Prognostic factors for outcomes in previous studies include the site of arterial occlusion, initial clinical status, time to thrombolysis, presence of symptomatic hemorrhage, or recanalization achievement. ${ }^{11-15}$ On the basis of the evaluation of predictors of 
Table 2: Comparisons between patients who had favorable and unfavorable outcomes 3 months after recanalization

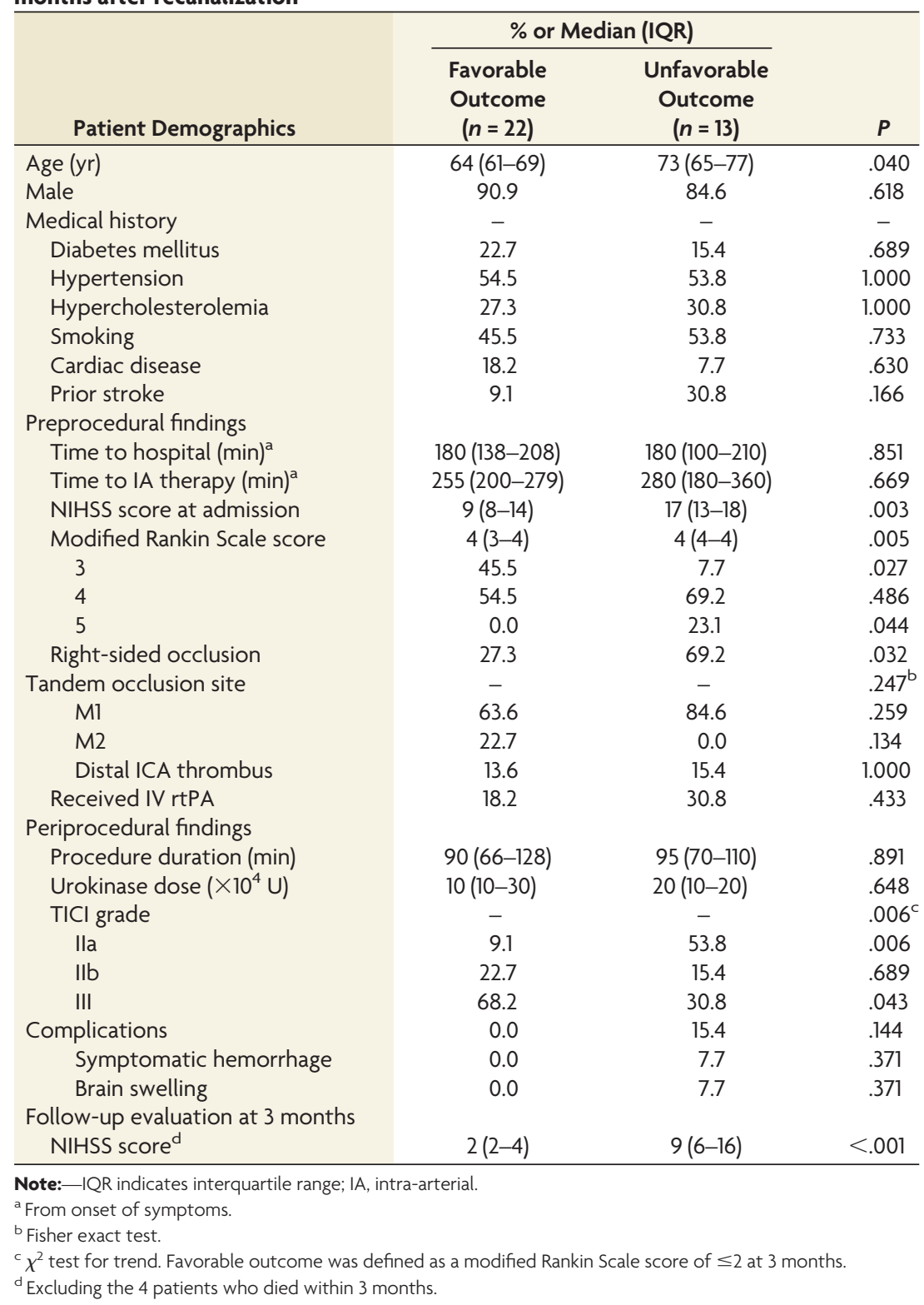

sion after revascularization in acute symptomatic ICA occlusion. We suggest that adequate distal filling of cerebral arteries is a simple and critical predictor of good outcome in acute symptomatic ICA occlusion.

There were no serious procedure-related complications such as dissection or vessel rupture in our study. In 1 patient, a symptomatic intracerebral hemorrhage was observed on CT 24 hours after the procedure. Also, 1 patient underwent decompressive surgery due to brain swelling and edema. Most interesting, we observed a lower mortality rate of $11 \%$ in our series in contrast to the study by Nedeltchev et al $(20 \%) .{ }^{5}$ We hypothesize that the use of new devices in our study and technical advances that have occurred since the time of the study of Nedeltchev et al, such as mechanical clot disruption and the mechanical thrombectomy device, most likely contributed to this rate.

The present study had a number of limitations. First, it was retrospective, so it was prone to selection and other biases. Second, we used multiple thrombectomy techniques for treatment of tandem occlusion, such as pharmacologic thrombolysis, mechanical clot disruption, or a mechanical thrombectomy device, making it difficult to determine the effect of the constituent techniques. Third, we did not have a control group with which to compare outcome rates. Last, we did not perform perfusion imaging in all patients before revascularization to quantify the ischemic area.

\section{CONCLUSIONS}

Our study showed that endovascular treatment by carotid artery stent placement and

Table 3: Multivariate logistic regression results for independent predictors of an unfavorable outcome after 3 months ${ }^{\text {a }}$

\begin{tabular}{|c|c|c|c|c|}
\hline \multirow[b]{2}{*}{ Predictor } & \multicolumn{2}{|c|}{ Univariate } & \multicolumn{2}{|c|}{ Multivariate } \\
\hline & OR $(95 \% \mathrm{CI})$ & $P$ & OR (95\% CI) & $P$ \\
\hline $\begin{array}{l}\text { NIHSS score at admission } \\
\text { (per unit change) }\end{array}$ & $1.4(1.1-1.7)$ & .003 & $1.8(1.1-3.1)$ & .022 \\
\hline TICI grade Ila & 11.7 (1.9-71.8) & .006 & $458(3.4-627)$ & .015 \\
\hline Right-sided occlusion & $6.0(1.3-27.0)$ & .032 & $31.9(1.1-9.1)$ & .043 \\
\hline
\end{tabular}

Note:-OR indicates odds ratio; $\mathrm{Cl}$, confidence interval.

a Variables were chosen by backward selection. Univariate columns show results for each variable fit separately. Multivariate columns show results from a single model fitting all variables simultaneously.

good outcome, our results indicate that better outcomes were related to younger age, lower initial NIHSS scores, left-sided occlusions, and TICI grades IIb or III after revascularization. The latter 3 were selected as independent predictors during multivariate analysis. Also, Suh et $\mathrm{al}^{14}$ reported that better short- and longterm outcomes were related to the degree of distal residual occlu-
IAT seems to improve outcome in patients with acute stroke caused by tandem occlusion of the MCA and/or distal ICA that results from ICA occlusion. Stroke severity, degree of successful revascularization, and the side of ischemia were found to independently predict functional outcomes at 3 months after treatment.

\section{REFERENCES}

1. Thanvi B, Robinson T. Complete occlusion of extracranial internal carotid artery: clinical features, pathophysiology, diagnosis and management. Postgrad Med J 2007;83:95-99

2. Meves SH, Muhs A, Federlein J, et al. Recanalization of acute symptomatic occlusions of the internal carotid artery. J Neurol 2002;249: $188-92$

3. Adams HP Jr, Bendixen BH, Leira E, et al. Antithrombotic treatment of ischemic stroke among patients with occlusion or severe stenosis of the internal carotid artery: a report of the Trial of Org 10172 in Acute Stroke Treatment (TOAST). Neurology 1999;53:122-25

4. Jovin TG, Gupta R, Uchino K, et al. Emergent stenting of extracra- 
nial internal carotid artery occlusion in acute stroke has a high revascularization rate. Stroke 2005;36:2426-30

5. Nedeltchev K, Brekenfeld C, Remonda L, et al. Internal carotid artery stent implantation in 25 patients with acute stroke: preliminary results. Radiology 2005;37:1029-37

6. Imai $\mathrm{K}$, Mori $\mathrm{T}$, Izumoto $\mathrm{H}$, et al. Emergency carotid artery stent placement in patients with acute ischemic stroke. AJNR Am J Neuroradiol 2005;26:1249-58

7. Lee HO, Koh EJ, Choi HY. Emergency carotid artery stent insertion for acute ICA occlusion. J Korean Neurosurg Soc 2010;47:428-32

8. Kao HL, Lin MS, Wang CS, et al. Feasibility of endovascular recanalization for symptomatic cervical internal carotid artery occlusion. J Am Coll Cardiol 2007;49:765-71

9. Mourand I, Brunel H, Vendrell JF, et al. Endovascular stent-assisted thrombolysis in acute occlusive carotid artery dissection. Neuroradiology 2010;52:135-40

10. Rubiera M, Ribo M, Delgado-Mederos R, et al. Tandem internal carotid artery/middle cerebral artery occlusion: an independent pre- dictor of poor outcome after systemic thrombolysis. Stroke 2006;37: 2301-05

11. Linfante I, Linas RH, Selim M, et al. Clinical and vascular outcome in internal carotid artery versus middle cerebral artery occlusions after intravenous tissue plasminogen activator. Stroke 2002;33:2066-71

12. Ernst R, Pancioli A, Tomsick K, et al. Combined intravenous and intra-arterial recombinant tissue plasminogen activator in acute ischemic stroke. Stroke 2000;31:2552-57

13. Zaidat OO, Suarez JI, Santillan C, et al. Response to intra-arterial and combined intravenous and intra-arterial thrombolytic therapy in patients with distal internal carotid artery occlusion. Stroke 2002; 33:1821-26

14. Suh DC, Kim JK, Choi CG, et al. Prognostic factors for neurologic outcome after endovascular revascularization of acute symptomatic occlusion of the internal carotid artery. AJNR Am J Neuroradiol 2007;28:1167-71

15. Rha JH, Saver IL. The impact of recanalization on ischemic stroke outcome: a meta-analysis. Stroke 2007;38:967-73 